\title{
Global Behavior for a Diffusive Predator-Prey Model with Stage Structure and Nonlinear Density Restriction-II: The Case in $\mathbb{R}^{1}$
}

\author{
Rui Zhang, ${ }^{1,2}$ Ling Guo, ${ }^{1}$ and Shengmao Fu $^{1}$ \\ ${ }^{1}$ Department of Mathematics, Northwest Normal University, Lanzhou 730070, China \\ ${ }^{2}$ Department of Mathematics, Lanzhou Jiaotong University, Lanzhou 730070, China
}

Correspondence should be addressed to Shengmao Fu, fusm@nwnu.edu.cn

Received 2 April 2009; Accepted 31 August 2009

Recommended by Wenming Zou

A Holling type III predator-prey model with self- and cross-population pressure is considered. Using the energy estimate and Gagliardo-Nirenberg-type inequalities, the existence and uniform boundedness of global solutions to the model are dicussed. In addition, global asymptotic stability of the positive equilibrium point for the model is proved by Lyapunov function.

Copyright (C) 2009 Rui Zhang et al. This is an open access article distributed under the Creative Commons Attribution License, which permits unrestricted use, distribution, and reproduction in any medium, provided the original work is properly cited.

\section{Introduction}

This paper is a continuation of Part I [1]. In Section 3 of Part I, using the energy estimate and bootstrap arguments, the global existence of solutions for a Holling type III cross-diffusion predator-prey model with stage-structure has been discussed when the space dimension be less than 6 . However, to obtain the $L^{\infty}$ estimate for the population density $w$ of predator species, there is not cross-diffusion for $w$ in Part I.

All diffusive predator-prey systems behave, more or less, in the same way, for both semilinear and cross-diffusive models, at least for small values of the cross diffusivities. Consequently, all the available information for linear diffusive models is essential to realize the behavior of the most complicated cross-diffusive systems [2-17].

In this paper, we consider the following cross-diffusion system:

$$
\begin{aligned}
& u_{t}=\left(d u+\alpha_{11} u^{2}+\alpha_{12} u v+\alpha_{13} u w\right)_{x x}+\beta v-a u-b u^{2}-c u^{3}-\frac{u^{2} w}{1+u^{2}} \\
& v_{t}=\left(d v+\alpha_{21} u v+\alpha_{22} v^{2}+\alpha_{23} v w\right)_{x x}+u-v, \quad 0<x<1, t>0
\end{aligned}
$$




$$
\begin{aligned}
w_{t} & =\left(d_{3} w+\alpha_{31} u w+\alpha_{32} v w+\alpha_{33} w^{2}\right)_{x x}-k w-\gamma w^{2}+\frac{\alpha u^{2} w}{1+u^{2}}, \\
u_{x}(x, t) & =v_{x}(x, t)=w_{x}(x, t)=0, \quad x=0,1, t>0, \\
u(x, 0) & =u_{0}(x), \quad v(x, 0)=v_{0}(x), \quad w(x, 0)=w_{0}(x), \quad 0<x<1,
\end{aligned}
$$

where $d, d_{3}, \alpha_{i j}(i, j=1,2,3), \alpha, \beta, \gamma, a, b, c$, and $k$ are positive constants. Also, $d, d_{3}$ are linear diffusion coefficients of $u, v, w$, respectively, while $\alpha_{i i}(i=1,2,3)$ are referred as self-diffusion pressures, and $\alpha_{i j}(i \neq j, i, j=1,2,3)$ are cross-diffusion pressures. If $\alpha_{12}=\alpha_{21}=\alpha_{23}=\alpha_{31}=$ $\alpha_{32}=0$, then (1.1) reduces to the system (1.4) of Part I.

Recently, the work in [18-20] studied the existence, uniform boundedness, and uniform convergence of global solutions for the Lotka-Volterra cross-diffusion models without stage-structure in the case that the space dimension $n=1$. In this paper, we consider mainly the existence and uniform boundedness of global solutions for the model (1.1) with nonlinear density restriction and stage-structure. Moreover, global asymptotic stability of the positive equilibrium point for (1.1) is proved by an important lemma of [21]. The proof is complete and complement the uniform convergence theorem in [18-20].

\section{Global Existence and Uniform Boundedness}

For simplicity, denote $|\cdot|_{k, p}=\|\cdot\|_{W_{p}^{k}(0,1)},|\cdot|_{p}=\|\cdot\|_{L^{p}(0,1)}$. The local existence result of solutions to (1.1) is an immediate consequence of a series of papers [22, 23] by Amann. Roughly speaking, if $u_{0}, v_{0}, w_{0} \in W_{p}^{1}(0,1), p>1$, then (1.1) has a unique nonnegative solution $u, v, w \in C\left([0, T), W_{p}^{1}(0,1)\right) \cap C^{\infty}\left((0, T), C^{\infty}(0,1)\right)$, where $T \leq+\infty$ is the maximal existence time for the solution. If $(u, v, w)$ satisfies

$$
\sup \left\{|u(\cdot, t)|_{1, p^{\prime}}|v(\cdot, t)|_{1, p}|w(\cdot, t)|_{1, p}: 0<t<T\right\}<\infty
$$

then $T=+\infty$. If, in addition, $u_{0}, v_{0}, w_{0} \in W_{p}^{2}(0,1)$, then $u, v, w \in C\left([0, \infty), W_{p}^{2}(0,1)\right)$.

The main result in this section is as follows.

Theorem 2.1. Let $u_{0}, v_{0}, w_{0} \in W_{2}^{2}(0,1),(u, v, w)$ is the unique nonnegative solution of (1.1) in its maximal existence interval $[0, T)$. Assume that

$$
\begin{aligned}
& 8 \alpha_{11} \alpha_{21} \alpha_{31}>\alpha_{21} \alpha_{13}^{2}+\alpha_{12}^{2} \alpha_{31} \\
& 8 \alpha_{12} \alpha_{22} \alpha_{32}>\alpha_{32} \alpha_{21}^{2}+\alpha_{23}^{2} \alpha_{12} \\
& 8 \alpha_{13} \alpha_{23} \alpha_{33}>\alpha_{23} \alpha_{31}^{2}+\alpha_{32}^{2} \alpha_{13}
\end{aligned}
$$


Then there exists $t_{0}>0$ and positive constants $M, M^{\prime}$ which depend on $d, d_{3}, \alpha_{i j}(i, j=1,2,3)$, $\beta, a, b, c, k, \gamma, \alpha$, such that

$$
\begin{gathered}
\sup \left\{|u(\cdot, t)|_{1,2},|v(\cdot, t)|_{1,2},|w(\cdot, t)|_{1,2}: t \in\left(t_{0}, T\right)\right\} \leq M^{\prime}, \\
\max \left\{u(x, t), v(x, t), w(x, t): 0 \leq x \leq 1, t_{0} \leq t<T\right\} \leq M,
\end{gathered}
$$

and $T=+\infty$. In particular, if $d, d_{3} \geq 1, d_{3} / d \in[\underline{d}, \bar{d}]$, where $\underline{d} \leq 1$ and $\bar{d}$ are positive constants, then $M^{\prime}, M$ depend on $\underline{d}, \bar{d}$, but do not depend on $d, d_{3} \geq 1$.

The following Gagliardo-Nirenberg-type inequalities and corresponding corollary play an importance role in the proof of Theorem 2.1.

Theorem 2.2 (see [18]). Let $\Omega \subset R^{n}$ be a bounded domain with $\partial \Omega \in C^{m}$. For every function $u \in W_{r}^{m}(\Omega), 1 \leq q, r \leq \infty$, the derivative $D^{j} u(0 \leq j<m)$ satisfies the inequality

$$
\left|D^{j} u\right|_{p} \leq C\left(\left|D^{m} u\right|_{r}^{a}|u|_{q}^{1-a}+|u|_{q}\right)
$$

provided one of the following three conditions is satisfied: (1) $r \leq q$, (2) $0<n(r-q) / m r q<1$, or (3) $n(r-q) / m r q=1$, and $m-n / q$ is not a nonnegative integer, where $1 / p=j / n+a(1 / r-m / n)+$ $(1-a) / q$, for all $a \in[j / m, 1)$, and the positive constant $C$ depends on $n, m, j, q, r, a$.

Corollary 2.3. There exists a positive constant $C$ such that

$$
\begin{gathered}
|u|_{2} \leq C\left(\left|u_{x}\right|_{2}^{1 / 3}|u|_{1}^{2 / 3}+|u|_{1}\right), \quad \forall u \in W_{2}^{1}(0,1), \\
|u|_{4} \leq C\left(\left|u_{x}\right|_{2}^{1 / 2}|u|_{1}^{1 / 2}+|u|_{1}\right), \quad \forall u \in W_{2}^{1}(0,1), \\
|u|_{7 / 2} \leq C\left(\left|u_{x}\right|_{2}^{10 / 21}|u|_{1}^{11 / 21}+|u|_{1}\right), \quad \forall u \in W_{2}^{1}(0,1), \\
\left|u_{x}\right|_{2} \leq C\left(\left|u_{x x}\right|_{2}^{3 / 5}|u|_{1}^{2 / 5}+|u|_{1}\right), \quad \forall u \in W_{2}^{2}(0,1) .
\end{gathered}
$$

For simplicity, denote that $C$ is Sobolev embedding constant or other kind of absolute constant. $A_{j}, B_{j}, C_{j}$ are some positive constants which depend on $\alpha_{i j}(i, j=1,2,3), \beta, a, b, c, k, \gamma, \alpha$. Also, $K_{j}$ are positive constants which depend on $\alpha_{i j}(i, j=1,2,3), \beta, a, b, c, k, \gamma, \alpha, d, d_{3}$. When $d, d_{3} \geq 1, K_{j}$ do not depend on $d, d_{3}$, but on $\underline{d}, \bar{d}$.

Proof of Theorem 2.1

Step 1. Estimate $|u|_{1},|v|_{1},|w|_{1}$. Firstly, taking integration of the first and second equations in (2.7) over the domain $[0,1]$, respectively, and combining the two integration equalities 
linearly, we have

$$
\frac{d}{d t} \int_{0}^{1}[u+(a+\beta) v] d x \leq-a \int_{0}^{1} v d x+\int_{0}^{1}\left(\beta u-b u^{2}\right) d x
$$

From Young inequality and Hölder inequality, we can see

$$
\frac{d}{d t} \int_{0}^{1}[u+(a+\beta) v] d x \leq C_{1}-\frac{a}{a+\beta} \int_{0}^{1}[u+(a+\beta) v] d x
$$

where $C_{1}=(1 / 4 b)(\beta+a /(a+\beta))^{2}$. From which it follows that there exists a constant $\tau_{0}>0$, such that

$$
\int_{0}^{1} u d x, \int_{0}^{1} v d x \leq M_{0}, \quad t \geq \tau_{0}
$$

where $M_{0}=\left(2 C_{1}(a+\beta) / a\right) \max \left\{(a+\beta)^{-1}, 1\right\}$.

Secondly, taking integration of the third equations in (2.7) over domain $[0,1]$, we have

$$
\frac{d}{d t} \int_{0}^{1} w d x \leq(\alpha-k) \int_{0}^{1} w d x-\gamma\left(\int_{0}^{1} w d x\right)^{2} .
$$

This implies that there exists a constant $\widetilde{\tau}_{0}>0$, such that

$$
\int_{0}^{1} w d x \leq \frac{2|\alpha-k|}{r}, \quad t \geq \widetilde{\tau}_{0}
$$

Let $M_{1}=\max \left\{M_{0},(2|\alpha-k|) / \gamma\right\}, \tau_{1}=\max \left\{\tau_{0}, \widetilde{\tau}_{0}\right\}$. Then

$$
\int_{0}^{1} u d x, \int_{0}^{1} v d x, \int_{0}^{1} w d x \leq M_{1}, \quad t \geq \tau_{1}
$$

Moreover, there exists a positive constant $M_{1}^{\prime}$ which depends on $\beta, a, b, c, k, \gamma, \alpha$ and the $L^{1}$ norm of $u_{0}, v_{0}, w_{0}$, such that

$$
\int_{0}^{1} u d x, \int_{0}^{1} v d x, \int_{0}^{1} w d x \leq M_{1}^{\prime}, \quad t \geq 0
$$


Step 2. estimate $|u|_{2},|v|_{2}$ and $|w|_{2}$. Multiplying the first three inequalities of Corollary 2.3 by $u, v, w$, respectively, and integrating over $[0,1]$, we have

$$
\begin{aligned}
& \frac{1}{2} \frac{d}{d t} \int_{0}^{1} u^{2} d x \\
& \quad \leq-d \int_{0}^{1} u_{x}^{2} d x-\int_{0}^{1}\left[\left(2 \alpha_{11} u+\alpha_{12} v+\alpha_{13} w\right) u_{x}^{2}+\alpha_{12} u u_{x} v_{x}+\alpha_{13} u u_{x} w_{x}\right] d x+\beta \int_{0}^{1} u v d x \\
& \frac{1}{2} \frac{d}{d t} \int_{0}^{1} v^{2} d x \\
& \quad \leq-d \int_{0}^{1} v_{x}^{2} d x-\int_{0}^{1}\left[\left(\alpha_{21} u+2 \alpha_{22} v+\alpha_{23} w\right) v_{x}^{2}+\alpha_{21} v u_{x} v_{x}+\alpha_{23} v v_{x} w_{x}\right] d x+\int_{0}^{1} u v d x \\
& \frac{1}{2} \frac{d}{d t} \int_{0}^{1} w^{2} d x \\
& \quad \leq-d_{3} \int_{0}^{1} w_{x}^{2} d x-\int_{0}^{1}\left[\left(\alpha_{31} u+\alpha_{32} v+2 \alpha_{33} w\right) w_{x}^{2}+\alpha_{31} w u_{x} w_{x}+\alpha_{32} w v_{x} w_{x}\right] d x
\end{aligned}
$$

Let $d^{\prime}=\min \left\{d, d_{3}\right\}$. By the above three inequalities and Young inequality, we have

$$
\begin{aligned}
& \frac{1}{2} \frac{d}{d t} \int_{0}^{1}\left(u^{2}+v^{2}+w^{2}\right) d x \\
& \quad \leq-d^{\prime} \int_{0}^{1}\left(u_{x}^{2}+v_{x}^{2}+w_{x}^{2}\right) d x-\int_{0}^{1} q\left(u_{x}, v_{x}, w_{x}\right) d x+\left(\frac{\beta+1}{2}+\alpha\right) \int_{0}^{1}\left(u^{2}+v^{2}+w^{2}\right) d x
\end{aligned}
$$

where

$$
\begin{aligned}
q\left(u_{x}, v_{x}, w_{x}\right)= & \left(2 \alpha_{11} u+\alpha_{12} v+\alpha_{13} w\right) u_{x}^{2}+\left(\alpha_{21} u+2 \alpha_{22} v+\alpha_{23} w\right) v_{x}^{2}+\left(\alpha_{31} u+\alpha_{32} v+2 \alpha_{33} w\right) w_{x}^{2} \\
& +\left(\alpha_{12} u+\alpha_{21} v\right) u_{x} v_{x}+\left(\alpha_{13} u+\alpha_{31} w\right) u_{x} w_{x}+\left(\alpha_{23} v+\alpha_{32} w\right) v_{x} w_{x}
\end{aligned}
$$

is quadratic form of $u_{x}, v_{x}, w_{x}$. It is not hard to verify that $q\left(u_{x}, v_{x}, w_{x}\right)$ is positive definite if (2.2) holds. Moreover, if (2.2) holds, then

$$
\frac{1}{2} \frac{d}{d t} \int_{0}^{1}\left(u^{2}+v^{2}+w^{2}\right) d \leq-d^{\prime} \int_{0}^{1}\left(u_{x}^{2}+v_{x}^{2}+w_{x}^{2}\right) d x+\left(\frac{\beta+1}{2}+\alpha\right) \int_{0}^{1}\left(u^{2}+v^{2}+w^{2}\right) d x
$$

Now we proceed in the following two cases.

(i) It holds that $t \geq \tau_{1}$. By (2.6) and (2.15), we have $\int_{0}^{1} u_{x}^{2} d x \geq\left(1 / C M_{1}^{4}\right)\left(\int_{0}^{1} u^{2} d x\right)^{3}-M_{1}^{2}$, 
and

$$
-d^{\prime} \int_{0}^{1}\left(u_{x}^{2}+v_{x}^{2}+w_{x}^{2}\right) d x \leq 3 d^{\prime} M_{1}^{2}-C_{2} d^{\prime}\left[\int_{0}^{1}\left(u^{2}+v^{2}+w^{2}\right) d x\right]^{3} .
$$

By (2.19) and (2.20), we can see that

$$
\begin{aligned}
& \frac{1}{2} \frac{d}{d t} \int_{0}^{1}\left(u^{2}+v^{2}+w^{2}\right) d x \\
& \quad \leq-C_{2} d^{\prime}\left[\int_{0}^{1}\left(u^{2}+v^{2}+w^{2}\right) d x\right]^{3}+\left(\frac{\beta+1}{2}+\alpha\right) \int_{0}^{1}\left(u^{2}+v^{2}+w^{2}\right) d x+3 d^{\prime} M_{1}^{2}
\end{aligned}
$$

Thus, there exists positive constants $\tau_{2}>\tau_{1}$ and $M_{2}$ depending on $d, d_{3}, \beta, a, b, c, k, \gamma, \alpha$, such that

$$
\int_{0}^{1} u^{2} d x, \int_{0}^{1} v^{2} d x, \int_{0}^{1} w^{2} d x \leq M_{2}^{\prime}, \quad t \geq \tau_{2}
$$

Since the zero point of the right-hand side in (2.21) can be estimated by positive constants independent of $d^{\prime}$, when $d^{\prime} \geq 1$. Thus $M_{2}$ do not depend on $d^{\prime} \geq 1$.

(ii) $t \geq 0$. Repeating estimates in (i) by $(2.9)^{\prime}$, we can obtain that there exists a positive constant $M_{2}^{\prime}$ depending on $d, d_{3}, \beta, a, b, c, k, \gamma, \alpha$ and the $L^{1}, L^{2}$-norm of $u_{0}, v_{0}, w_{0}$, such that

$$
\int_{0}^{1} u^{2} d x, \int_{0}^{1} v^{2} d x, \int_{0}^{1} w^{2} d x \leq M_{2}^{\prime}, \quad t \geq 0
$$

when $d^{\prime} \geq 1, M_{1}^{\prime}$ is independent of $d^{\prime}$.

Step 3. Estimate $\left|u_{x}\right|_{2},\left|v_{x}\right|_{2},\left|w_{x}\right|_{2}$. Introduce the scaling that

$$
\tilde{u}=\frac{u}{d_{1}}, \quad \tilde{v}=\frac{v}{d_{1}}, \quad \tilde{w}=\frac{w}{d_{1}}, \quad \tilde{t}=d_{1} t
$$

denote $\eta=d_{3} / d$, and redenote $\tilde{u}, \widetilde{v}, \widetilde{w}, \tilde{t}$ by $u, v, w, t$, respectively. Then (2.7) reduces to

$$
\begin{aligned}
u_{t} & =P_{x x}+f(u, v, w), \quad 0<x<1, t>0, \\
v_{t} & =Q_{x x}+g(u, v, w), \quad 0<x<1, t>0, \\
w_{t} & =R_{x x}+h(u, v, w), \quad 0<x<1, t>0, \\
u_{x}(x, t) & =v_{x}(x, t)=w_{x}(x, t)=0, \quad x=0,1, t>0, \\
u(x, 0) & =\tilde{u}_{0}(x), \quad v(x, 0)=\widetilde{v}_{0}(x), \quad w(x, 0)=\widetilde{w}_{0}(x), \quad 0<x<1,
\end{aligned}
$$


where $P=u+\alpha_{11} u^{2}+\alpha_{12} u v+\alpha_{13} u w, Q=v+\alpha_{21} u v+\alpha_{22} v^{2}+\alpha_{23} v w, R=\eta w+\alpha_{31} u w+\alpha_{32} v w+$ $\alpha_{33} w^{2}, f(u, v, w)=\beta d^{-1} v-a d^{-1} u-b u^{2}-c d u^{3}-\left(d u^{2} w /\left(1+d^{2} u^{2}\right)\right), g(u, v, w)=d^{-1}(u-v)$, $h(u, v, w)=-k d^{-1} w-r w^{2}+\left(\alpha d u^{2} w /\left(1+d^{2} u^{2}\right)\right)$. We still proceed in following two cases.

(i) It holds that $t \geq \tau_{2}^{*}=d \tau_{2}$. From (2.15) and (2.22), we can easily obtain that

$$
\begin{gathered}
\int_{0}^{1} u d x, \int_{0}^{1} v d x, \int_{0}^{1} w d x \leq M_{1} d^{-1}, \\
\int_{0}^{1} u^{2} d x, \int_{0}^{1} v^{2} d x, \int_{0}^{1} w^{2} d x \leq M_{2} d^{-2}, \\
|P|_{1},|Q|_{1},|R|_{1} \leq D K_{1} d^{-1},
\end{gathered}
$$

where $K_{1}=(2+\eta)+M_{2} d^{-2}, D=\max \left\{M_{1}, \alpha_{11}+\alpha_{12}+\alpha_{13}, \alpha_{21}+\alpha_{22}+\alpha_{23}, \alpha_{31}+\alpha_{32}+\alpha_{33}\right\}$.

Multiply the first three equations in (2.24) by $P_{t}, Q_{t}, R_{t}$ and integrate them over [0,1], respectively, then adding up the three new equations, we have

$$
\begin{aligned}
\frac{1}{2} \bar{y}^{\prime}(t) \leq & -\int_{0}^{1} u_{t}^{2} d x-\int_{0}^{1} v_{t}^{2} d x-\eta \int_{0}^{1} w_{t}^{2} d x-\int_{0}^{1} q\left(u_{t}, v_{t}, w_{t}\right) d x \\
& +\int_{0}^{1}\left[\left(1+2 \alpha_{11} u+\alpha_{12} v+\alpha_{13} w\right) u_{t} f+\alpha_{12} u v_{t} f+\alpha_{13} u w_{t} f\right] d x \\
& +\int_{0}^{1}\left[\alpha_{21} v u_{t} g+\left(1+\alpha_{21} u+2 \alpha_{22} v+\alpha_{23} w\right) v_{t} g+\alpha_{23} v w_{t} g\right] d x \\
& +\int_{0}^{1}\left[\alpha_{31} w u_{t} h+\alpha_{32} w v_{t} h+\left(\eta+\alpha_{31} u+\alpha_{32} v+2 \alpha_{33} w\right) w_{t} h\right] d x,
\end{aligned}
$$

where $\bar{y}=\int_{0}^{1}\left(P_{x}^{2}+Q_{x}^{2}+R_{x}^{2}\right) d x$. It is not hard to verify by (2.4) that there exists a positive constant $C_{3}$ depending only on $\alpha_{i j}(i, j=1,2,3)$, such that

$$
q\left(u_{t}, v_{t}, w_{t}\right) \geq C_{3}(u+v+w)\left(u_{t}^{2}+v_{t}^{2}+w_{t}^{2}\right) .
$$

Thus,

$$
\begin{aligned}
\frac{1}{2} \bar{y}^{\prime}(t) & \leq-\int_{0}^{1} u_{t}^{2} d x-\int_{0}^{1} v_{t}^{2} d x-\eta \int_{0}^{1} w_{t}^{2} d x-C_{3} \int_{0}^{1}(u+v+w)\left(u_{t}^{2}+v_{t}^{2}+w_{t}^{2}\right) d x \\
& +\int_{0}^{1}\left(1+2 \alpha_{11} u+\alpha_{12} v+\alpha_{13} w\right) u_{t} f d x+\int_{0}^{1}\left(1+\alpha_{21} u+2 \alpha_{22} v+\alpha_{23} w\right) v_{t} g d x \\
& +\int_{0}^{1}\left(\eta+\alpha_{31} u+\alpha_{32} v+2 \alpha_{33} w\right) w_{t} h d x+\int_{0}^{1} \alpha_{12} u v_{t} f d x+\int_{0}^{1} \alpha_{13} u w_{t} f d x \\
& +\int_{0}^{1} \alpha_{21} v u_{t} g d x+\int_{0}^{1} \alpha_{23} v w_{t} g d x+\int_{0}^{1} \alpha_{31} w u_{t} h d x+\int_{0}^{1} \alpha_{32} w v_{t} h d x
\end{aligned}
$$


8

Boundary Value Problems

Using Young inequality, Hölder inequality and (2.24), we can obtain the following estimates:

$$
\begin{aligned}
& \int_{0}^{1} u^{3} d x \leq\left(\int_{0}^{1} u^{7} d x\right)^{1 / 5}\left(\int_{0}^{1} u^{2} d x\right)^{4 / 5} \leq M_{2}^{4 / 5} d^{-8 / 5}\left(\int_{0}^{1} u^{7} d x\right)^{1 / 5}, \\
& \int_{0}^{1} u^{4} d x \leq\left(\int_{0}^{1} u^{7} d x\right)^{2 / 5}\left(\int_{0}^{1} u^{2} d x\right)^{3 / 5} \leq M_{2}^{3 / 5} d^{-6 / 5}\left(\int_{0}^{1} u^{7} d x\right)^{2 / 5}, \\
& \int_{0}^{1} u^{5} d x \leq\left(\int_{0}^{1} u^{7} d x\right)^{3 / 5}\left(\int_{0}^{1} u^{2} d x\right)^{2 / 5} \leq M_{2}^{2 / 5} d^{-4 / 5}\left(\int_{0}^{1} u^{7} d x\right)^{3 / 5}, \\
& \int_{0}^{1} u^{6} d x \leq\left(\int_{0}^{1} u^{7} d x\right)^{4 / 5}\left(\int_{0}^{1} u^{2} d x\right)^{1 / 5} \leq M_{2}^{1 / 5} d^{-2 / 5}\left(\int_{0}^{1} u^{7} d x\right)^{4 / 5}, \\
& \int_{0}^{1} u v d x \leq\left(\int_{0}^{1} u^{2} d x\right)^{1 / 2}\left(\int_{0}^{1} v^{2} d x\right)^{1 / 2} \leq M_{2} d^{-2}, \\
& \int_{0}^{1} u^{2} v d x \leq\left(\int_{0}^{1} u^{7} d x\right)^{1 / 5}\left(\int_{0}^{1} u^{2} d x\right)^{3 / 10}\left(\int_{0}^{1} v^{2} d x\right)^{1 / 2} \leq M_{2}^{4 / 5} d^{-8 / 5}\left(\int_{0}^{1} u^{7} d x\right)^{1 / 5}, \\
& \int_{0}^{1} u^{3} v d x \leq\left(\int_{0}^{1} u^{7} d x\right)^{2 / 5}\left(\int_{0}^{1} u^{2} d x\right)^{1 / 10}\left(\int_{0}^{1} v^{2} d x\right)^{1 / 2} \leq M_{2}^{3 / 5} d^{-6 / 5}\left(\int_{0}^{1} u^{7} d x\right)^{2 / 5}, \\
& \int_{0}^{1} u^{6} v d x \leq \frac{6}{7} \int_{0}^{1} u^{7} d x+\frac{1}{7} \int_{0}^{1} v^{7} d x \leq \frac{6}{7} \int_{0}^{1}\left(u^{7}+v^{7}\right) d x, \\
& \int_{0}^{1} u^{4} v d x \leq \frac{1}{2} \int_{0}^{1} u^{2} v d x+\frac{1}{2} \int_{0}^{1} u^{6} v d x \leq \frac{1}{2} M_{2}^{4 / 5} d^{-8 / 5}\left(\int_{0}^{1} u^{7} d x\right)^{1 / 5}+\frac{3}{7} \int_{0}^{1}\left(u^{7}+v^{7}\right) d x, \\
& \int_{0}^{1} u u_{t} d x \leq \frac{1}{2 \epsilon} \int_{0}^{1} u d x+\frac{\epsilon}{2} \int_{0}^{1} u u_{t}^{2} d x \leq \frac{1}{2 \epsilon} M_{1} d^{-1}+\frac{\epsilon}{2} \int_{0}^{1} u u_{t}^{2} d x ， \\
& \int_{0}^{1} u^{2} u_{t} d x \leq \frac{1}{2 \epsilon} \int_{0}^{1} u^{3} d x+\frac{\epsilon}{2} \int_{0}^{1} u u_{t}^{2} d x \leq \frac{1}{2 \epsilon} M_{2}^{4 / 5} d^{-8 / 5}\left(\int_{0}^{1} u^{7} d x\right)^{1 / 5}+\frac{\epsilon}{2} \int_{0}^{1} u u_{t}^{2} d x, \\
& \int_{0}^{1} u^{3} u_{t} d x \leq \frac{1}{2 \epsilon} \int_{0}^{1} u^{5} d x+\frac{\epsilon}{2} \int_{0}^{1} u u_{t}^{2} d x \leq \frac{1}{2 \epsilon} M_{2}^{2 / 5} d^{-4 / 5}\left(\int_{0}^{1} u^{7} d x\right)^{3 / 5}+\frac{\epsilon}{2} \int_{0}^{1} u u_{t}^{2} d x, \\
& \int_{0}^{1} u^{4} u_{t} d x \leq \frac{1}{2 \epsilon} \int_{0}^{1} u^{7} d x+\frac{\epsilon}{2} \int_{0}^{1} u u_{t}^{2} d x, \\
& \int_{0}^{1} u v u_{t} d x \leq \frac{1}{2 \epsilon} \int_{0}^{1} u^{2} v d x+\frac{\epsilon}{2} \int_{0}^{1} v u_{t}^{2} d x \leq \frac{1}{2 \epsilon} M_{2}^{4 / 5} d^{-8 / 5}\left(\int_{0}^{1} u^{7} d x\right)^{1 / 5}+\frac{\epsilon}{2} \int_{0}^{1} v u_{t}^{2} d x, \\
& \int_{0}^{1} u^{2} v u_{t} d x \leq \frac{1}{2 \epsilon} \int_{0}^{1} u^{4} v d x+\frac{\epsilon}{2} \int_{0}^{1} v u_{t}^{2} d x
\end{aligned}
$$


Boundary Value Problems

9

$$
\begin{aligned}
& \frac{1}{4 \epsilon} M_{2}^{4 / 5} d^{-8 / 5}\left(\int_{0}^{1} u^{7} d x\right)^{1 / 5}+\frac{3}{14 \epsilon} \int_{0}^{1}\left(u^{7}+v^{7}\right) d x+\frac{\epsilon}{2} \int_{0}^{1} v u_{t}^{2} d x, \\
\int_{0}^{1} u^{3} v u_{t} d x & \leq \frac{1}{2 \epsilon} \int_{0}^{1} u^{6} v d x+\frac{\epsilon}{2} \int_{0}^{1} v u_{t}^{2} d x \leq \frac{3}{14 \epsilon} \int_{0}^{1}\left(u^{7}+v^{7}\right) d x+\frac{\epsilon}{2} \int_{0}^{1} v u_{t}^{2} d x .
\end{aligned}
$$

Applying the above estimates and Gagliardo-Nirenberg-type inequalities to the terms on the right-hand side of (2.28), we have

$$
\begin{aligned}
& -\int_{0}^{1} u_{t}^{2} d x \leq-\frac{1}{2} \int_{0}^{1} P_{x x}^{2} d x+\int_{0}^{1} f^{2} d x \\
& -\int_{0}^{1} v_{t}^{2} d x \leq-\frac{1}{2} \int_{0}^{1} Q_{x x}^{2} d x+\int_{0}^{1} g^{2} d x, \\
& -\eta \int_{0}^{1} w_{t}^{2} d x \leq-\frac{\eta}{2} \int_{0}^{1} R_{x x}^{2} d x+\eta \int_{0}^{1} h^{2} d x \\
& \int_{0}^{1} f^{2} d x \leq \beta^{2} d^{-2} \int_{0}^{1} v^{2} d x+a^{2} d^{-2} \int_{0}^{1} u^{2} d x+b^{2} \int_{0}^{1} u^{4} d x+2 b c d^{2} \int_{0}^{1} u^{5} d x+c^{2} d^{4} \int_{0}^{1} u^{6} d x \\
& +2 a b d^{-1} \int_{0}^{1} u^{3} d x+2 a c \int_{0}^{1} u^{4} d x+d^{-2} \int_{0}^{1} w^{2} d x \\
& +2 a d^{-2} \int_{0}^{1} u w d x+2 b d^{-1} \int_{0}^{1} u^{2} w d x+2 \int_{0}^{1} u^{3} w d x \\
& \leq\left(a^{2}+\beta+1+2 a\right) M_{2} d^{-4}+2 b(a+1) M_{2}^{3 / 5} d^{-13 / 5}\left(\int_{0}^{1} u^{7} d x\right)^{1 / 5} \\
& +\left(2 a c+b^{2}+2\right) M_{2}^{3 / 5} d^{-6 / 5}\left(\int_{0}^{1} u^{7} d x\right)^{2 / 5} \\
& +2 b c M_{2}^{2 / 5} d^{1 / 5}\left(\int_{0}^{1} u^{7} d x\right)^{3 / 5}+c^{2} M_{2}^{1 / 5} d^{8 / 5}\left(\int_{0}^{1} u^{7} d x\right)^{4 / 5}, \\
& \int_{0}^{1} g^{2} d x \leq d^{-2} \int_{0}^{1}\left(u^{2}+v^{2}\right) d x \leq 2 M_{2} d^{-4}, \\
& \eta \int_{0}^{1} h^{2} d x \leq d^{-2} \eta\left(k^{2}+\alpha^{2}\right) \int_{0}^{1} w^{2} d x+2 k d^{-1} \gamma \eta \int_{0}^{1} w^{3} d x+\gamma^{2} \eta \int_{0}^{1} w^{4} d x \\
& \leq \eta\left(k^{2}+\alpha^{2}\right) M_{2} d^{-4}+2 k \gamma \eta M_{2}^{4 / 5} d_{1}^{-13 / 5}\left(\int_{0}^{1} w^{7} d x\right)^{1 / 5} \\
& +\gamma^{2} \eta M_{2}^{3 / 5} d^{-6 / 5}\left(\int_{0}^{1} w^{7} d x\right)^{2 / 5} .
\end{aligned}
$$


10

Boundary Value Problems

Thus

$$
\begin{aligned}
& -\int_{0}^{1} u_{t}^{2} d x-\int_{0}^{1} v_{t}^{2} d x-\eta \int_{0}^{1} w_{t}^{2} d x \\
& \leq-\frac{1}{2} \int_{0}^{1} P_{x x}^{2} d x-\frac{1}{2} \int_{0}^{1} Q_{x x}^{2} d x-\frac{\eta}{2} \int_{0}^{1} R_{x x}^{2} d x+C_{4}(2+\eta) M_{2} d^{-4} \\
& \quad+C_{5} d^{-1}(1+\eta) M_{2}^{4 / 5} d^{-8 / 5}\left[\int_{0}^{1}\left(u^{7}+w^{7}\right) d x\right]^{1 / 5}+C_{6}(1+\eta) M_{2}^{3 / 5} d^{-6 / 5}\left[\int_{0}^{1}\left(u^{5}+w^{7}\right) d x\right]^{2 / 5} \\
& \quad+C_{7} M_{2}^{2 / 5} d^{1 / 5}\left(\int_{0}^{1} u^{7} d x\right)^{3 / 5}+C_{8} M_{2}^{1 / 5} d^{8 / 5}\left(\int_{0}^{1} u^{7} d x\right)^{4 / 5} .
\end{aligned}
$$

For the other terms on the right-hand side of (2.28), we have

$$
\begin{aligned}
\int_{0}^{1} u_{t} f d x \leq & \beta d^{-1}\left|\int_{0}^{1} u_{t} v d x\right|+a d^{-1}\left|\int_{0}^{1} u u_{t} d x\right|+b\left|\int_{0}^{1} u^{2} u_{t} d x\right| \\
& +c d\left|\int_{0}^{1} u^{3} u_{t} d x\right|+d^{-1}\left|\int_{0}^{1} w u_{t} d x\right| \\
\leq & \frac{\beta^{2}+a^{2}+1}{2 \epsilon} M_{1} d^{-3}+\frac{b^{2}}{2 \epsilon} M_{2}^{4 / 5} d^{-8 / 5}\left(\int_{0}^{1} u^{7} d x\right)^{1 / 5} \\
& +\frac{c^{2}}{2 \epsilon} M_{2}^{2 / 5} d^{6 / 5}\left(\int_{0}^{1} u^{7} d x\right)^{3 / 5}+\frac{3}{2} \epsilon \int_{0}^{1} u u_{t}^{2} d x+\frac{\beta d^{-1}}{2} \epsilon \int_{0}^{1} v u_{t}^{2} d x+\frac{1}{2} \epsilon \int_{0}^{1} w u_{t}^{2} d x, \\
\alpha_{0}^{1} u u_{t} f d x \leq & 2 \alpha_{11} \beta d^{-1}\left|\int_{0}^{1} u u_{t} v d x\right|+2 \alpha_{11} a d^{-1}\left|\int_{0}^{1} u^{2} u_{t} d x\right| \\
& +2 \alpha_{11}^{2} b\left|\int_{0}^{1} u^{3} u_{t} d x\right|+2 \alpha_{11} d c\left|\int_{0}^{1} u^{4} u_{t} d x\right|+2 \alpha_{11} d^{-1}\left|\int_{0}^{1} u u_{t} w d x\right| \\
\leq & \frac{\alpha_{11}^{2}\left(\beta^{2}+a^{2}+1\right)}{\epsilon} M_{2}^{4 / 5} d^{-18 / 5}\left(\int_{0}^{1} u^{7} d x\right)^{1 / 5}+\frac{\alpha_{11}^{2} b^{2}}{\epsilon} M_{2}^{2 / 5} d^{-4 / 5}\left(\int_{0}^{1} u^{7} d x\right)^{3 / 5} \\
& +\frac{\alpha_{11}^{2} c^{2}}{\epsilon} d^{2} \int_{0}^{1} u^{7} d x+3 \epsilon \int_{0}^{1} u u_{t}^{2} d x+\epsilon \int_{0}^{1} v u_{t}^{2} d x+\epsilon \int_{0}^{1} w u_{t}^{2} d x,
\end{aligned}
$$


Boundary Value Problems

11

$$
\begin{aligned}
& \alpha_{12} \int_{0}^{1} v u_{t} f d x \leq \alpha_{12} \beta d^{-1}\left|\int_{0}^{1} u_{t} v^{2} d x\right|+\alpha_{12} a d^{-1}\left|\int_{0}^{1} u v u_{t} d x\right|+\alpha_{12}^{2} b\left|\int_{0}^{1} u^{2} v u_{t} d x\right| \\
& +\alpha_{12} d c\left|\int_{0}^{1} u^{3} v u_{t} d x\right|+\alpha_{12} d^{-1}\left|\int_{0}^{1} v w u_{t} d x\right| \\
& \leq \frac{\alpha_{12}^{2}}{2 \epsilon}\left(a^{2} d^{-2}+\frac{b^{2}}{2}\right) M_{2}^{4 / 5} d^{-8 / 5}\left(\int_{0}^{1} u^{7} d x\right)^{1 / 5} \\
& +\frac{\alpha_{12}^{2}\left(\beta^{2}+1\right)}{2 \epsilon} M_{2}^{4 / 5} d^{-18 / 5}\left(\int_{0}^{1} v^{7} d x\right)^{1 / 5} \\
& +\frac{3 \alpha_{12}^{2}}{7 \epsilon}\left(\frac{b^{2}}{2}+c^{2} d^{2}\right) \int_{0}^{1}\left(u^{7}+v^{7}\right) d x+\frac{5}{2} \epsilon \int_{0}^{1} v u_{t}^{2} d x \\
& \alpha_{13} \int_{0}^{1} w u_{t} f d x \leq \alpha_{13} \beta d^{-1}\left|\int_{0}^{1} v w u_{t} d x\right|+\alpha_{13} a d^{-1}\left|\int_{0}^{1} u w u_{t} d x\right|+\alpha_{13}^{2} b\left|\int_{0}^{1} u^{2} w u_{t} d x\right| \\
& +\alpha_{13} d c\left|\int_{0}^{1} u^{3} w u_{t} d x\right|+\alpha_{13} d^{-1}\left|\int_{0}^{1} w^{2} u_{t} d x\right| \\
& \leq \frac{\alpha_{13}^{2}\left(a^{2} d^{-2}+b^{2} / 2\right)}{2 \epsilon} M_{2}^{4 / 5} d^{-8 / 5}\left(\int_{0}^{1} u^{7} d x\right)^{1 / 5} \\
& +\frac{\alpha_{13}^{2}}{2 \epsilon} M_{2}^{4 / 5} d^{-18 / 5}\left[\beta\left(\int_{0}^{1} v^{7} d x\right)^{1 / 5}+\left(\int_{0}^{1} w^{7} d x\right)^{1 / 5}\right] \\
& +\frac{3 \alpha_{13}^{2}}{7 \epsilon}\left(\frac{b^{2}}{2}+c^{2} d^{2}\right) \int_{0}^{1}\left(u^{7}+w^{7}\right) d x+\frac{5}{2} \epsilon \int_{0}^{1} w u_{t}^{2} d x, \\
& \int_{0}^{1} v_{t} g d x \leq d^{-1}\left|\int_{0}^{1} u v_{t} d x\right|+d^{-1}\left|\int_{0}^{1} v v_{t} d x\right| \leq \frac{M_{1} d^{-3}}{\epsilon}+\frac{\epsilon}{2} \int_{0}^{1}\left(u v_{t}^{2}+v v_{t}^{2}\right) d x, \\
& \alpha_{21} \int_{0}^{1} u v_{t} g d x \leq \alpha_{21} d^{-1}\left|\int_{0}^{1} u^{2} v_{t} d x\right|+\alpha_{21} d^{-1}\left|\int_{0}^{1} u v v_{t} d x\right| \\
& \leq \frac{\alpha_{21}^{2}}{\epsilon} M_{2}^{4 / 5} d^{-18 / 5}\left(\int_{0}^{1} u^{7} d x\right)^{1 / 5}+\frac{\epsilon}{2} \int_{0}^{1}\left(u v_{t}^{2}+v v_{t}^{2}\right) d x, \\
& 2 \alpha_{22} \int_{0}^{1} v v_{t} g d x \leq 2 \alpha_{22} d^{-1}\left|\int_{0}^{1} u v v_{t} d x\right|+2 \alpha_{22} d^{-1}\left|\int_{0}^{1} v^{2} v_{t} d x\right| \\
& \leq \frac{\alpha_{22} \int_{0}^{1} v v_{t}^{2} d x}{\epsilon} M_{2}^{4 / 5} d^{-18 / 5}\left[\left(\int_{0}^{1} u^{7} d x\right)^{1 / 5}+\left(\int_{0}^{1} v^{7} d x\right)^{1 / 5}\right]+\epsilon \int_{0}^{1} v v_{t}^{2} d x, \\
& \alpha_{23} \int_{0}^{1} w v_{t} g d x \leq \alpha_{23} d^{-1}\left|\int_{0}^{1} u w v_{t} d x\right|+\alpha_{23} d^{-1}\left|\int_{0}^{1} v w v_{t} d x\right|
\end{aligned}
$$


12

Boundary Value Problems

$$
\begin{aligned}
& \leq \frac{\alpha_{23} \int_{0}^{1} v v_{t}^{2} d x}{2 \epsilon} M_{2}^{4 / 5} d^{-18 / 5}\left[\left(\int_{0}^{1} u^{7} d x\right)^{1 / 5}+\left(\int_{0}^{1} v^{7} d x\right)^{1 / 5}\right]+\epsilon \int_{0}^{1} w v_{t}^{2} d x, \\
& \eta \int_{0}^{1} w_{t} h d x \leq d^{-1} \eta(\alpha+k)\left|\int_{0}^{1} w w_{t} d x\right|+\gamma \eta\left|\int_{0}^{1} w^{2} w_{t} d x\right| \\
& \leq \frac{(\alpha+k)^{2}}{2 \epsilon} \eta^{2} d^{-3} M_{1}+\frac{\gamma^{2}}{2 \epsilon} \eta^{2} M_{2}^{4 / 5} d^{-8 / 5}\left(\int_{0}^{1} w^{7} d x\right)^{1 / 5}+\epsilon \eta \int_{0}^{1} w w_{t}^{2} d x, \\
& \alpha_{31} \int_{0}^{1} u w_{t} h d x \leq(\alpha+k) d^{-1} \alpha_{31}\left|\int_{0}^{1} u w w_{t} d x\right|+\alpha_{31} \gamma\left|\int_{0}^{1} u w^{2} w_{t} d x\right| \\
& \leq \frac{\alpha_{31}^{2}}{2 \epsilon}\left[(\alpha+k)^{2} d^{-2}+\frac{r^{2}}{2}\right] M_{2}^{4 / 5} d^{-8 / 5}\left[\left(\int_{0}^{1} u^{7} d x\right)^{1 / 5}+\left(\int_{0}^{1} w^{7} d x\right)^{1 / 5}\right] \\
& +\frac{3 \alpha_{31}^{2} \gamma^{2}}{14 \epsilon} \int_{0}^{1}\left(u^{7}+w^{7}\right) d x+\frac{1}{2} \epsilon \int_{0}^{1} u w_{t}^{2} d x+\frac{1}{2} \epsilon \int_{0}^{1} w w_{t}^{2} d x, \\
& \alpha_{32} \int_{0}^{1} v w_{t} h d x \leq(\alpha+k) d^{-1} \alpha_{32}\left|\int_{0}^{1} v w w_{t} d x\right|+\alpha_{32} \gamma\left|\int_{0}^{1} v w^{2} w_{t} d x\right| \\
& \leq \frac{\alpha_{32}^{2}}{2 \epsilon}\left[(\alpha+k)^{2} d^{-2}+\frac{r^{2}}{2}\right] M_{2}^{4 / 5} d^{-8 / 5}\left[\left(\int_{0}^{1} v^{7} d x\right)^{1 / 5}+\left(\int_{0}^{1} w^{7} d x\right)^{1 / 5}\right] \\
& +\frac{3 \alpha_{32}^{2}}{14 \epsilon} \gamma^{2} \int_{0}^{1}\left(v^{7}+w^{7}\right) d x+\frac{1}{2} \epsilon \int_{0}^{1} v w_{t}^{2} d x+\frac{1}{2} \epsilon \int_{0}^{1} w w_{t}^{2} d x, \\
& 2 \alpha_{33} \int_{0}^{1} w w_{t} h d x \leq 2(\alpha+k) d^{-1} \alpha_{33}\left|\int_{0}^{1} w^{2} w_{t} d x\right|+2 \alpha_{33} \gamma\left|\int_{0}^{1} w^{3} w_{t} d x\right| \\
& \leq \frac{(\alpha+k)^{2} \alpha_{33}^{2}}{\epsilon} M_{2}^{4 / 5} d^{-18 / 5}\left(\int_{0}^{1} w^{7} d x\right)^{1 / 5}+\frac{\alpha_{33}^{2} \gamma^{2}}{\epsilon} M_{2}^{2 / 5} d^{-4 / 5}\left(\int_{0}^{1} w^{7} d x\right)^{3 / 5} \\
& +\epsilon \int_{0}^{1} w w_{t}^{2} d x \\
& \alpha_{12} \int_{0}^{1} u v_{t} f d x \leq \alpha_{12} \beta d^{-1}\left|\int_{0}^{1} u v v_{t} d x\right|+\alpha_{12} a d^{-1}\left|\int_{0}^{1} u^{2} v_{t} d x\right|+\alpha_{12} b\left|\int_{0}^{1} u^{3} v_{t} d x\right| \\
& +\alpha_{12} c d\left|\int_{0}^{1} u^{4} v_{t} d x\right|+\alpha_{12} d^{-1}\left|\int_{0}^{1} u w v_{t} d x\right| \\
& \leq \frac{\alpha_{12}^{2}\left(\beta^{2}+a^{2}+1\right)}{2 \epsilon} M_{2}^{4 / 5} d^{-18 / 5}\left(\int_{0}^{1} u^{7} d x\right)^{1 / 5}+\frac{\alpha_{12}^{2} b^{2}}{2 \epsilon} M_{2}^{2 / 5} d^{-4 / 5}\left(\int_{0}^{1} u^{7} d x\right)^{3 / 5}
\end{aligned}
$$


Boundary Value Problems

13

$$
\begin{aligned}
& +\frac{\alpha_{12}^{2} c^{2}}{2 \epsilon} d^{2} \int_{0}^{1} u^{7} d x+\frac{\epsilon}{2}\left[3 \int_{0}^{1} u v_{t}^{2} d x+\int_{0}^{1} v v_{t}^{2} d x+\int_{0}^{1} w v_{t}^{2} d x\right], \\
& \alpha_{13} \int_{0}^{1} u w_{t} f d x \leq \alpha_{13} \beta d^{-1}\left|\int_{0}^{1} u v w_{t} d x\right|+\alpha_{13} a d^{-1}\left|\int_{0}^{1} u^{2} w_{t} d x\right|+\alpha_{13} b\left|\int_{0}^{1} u^{3} w_{t} d x\right| \\
& +\alpha_{13}^{3} c d\left|\int_{0}^{1} u^{4} w_{t} d x\right|+\alpha_{13} d^{-1}\left|\int_{0}^{1} u w w_{t} d x\right| \\
& \leq \frac{\alpha_{13}^{2}\left(\beta^{2}+a^{2}+1\right)}{2 \epsilon} M_{2}^{4 / 5} d^{-18 / 5}\left(\int_{0}^{1} u^{7} d x\right)^{1 / 5}+\frac{\alpha_{13}^{2} b^{2}}{2 \epsilon} M_{2}^{2 / 5} d^{-4 / 5}\left(\int_{0}^{1} u^{7} d x\right)^{3 / 5} \\
& +\frac{\alpha_{13}^{2} c^{2}}{2 \epsilon} d^{2} \int_{0}^{1} u^{7} d x+\frac{3}{2} \epsilon \int_{0}^{1} u w_{t}^{2} d x+\frac{1}{2} \epsilon \int_{0}^{1} v w_{t}^{2} d x+\frac{1}{2} \epsilon \int_{0}^{1} w w_{t}^{2} d x, \\
& \alpha_{21} \int_{0}^{1} v u_{t} g d x \leq \alpha_{21} d^{-1}\left|\int_{0}^{1} u v u_{t} d x\right|+\alpha_{21} d^{-1}\left|\int_{0}^{1} v^{2} u_{t} d x\right| \\
& \leq \frac{\alpha_{21}^{2}}{2 \epsilon} M_{2}^{4 / 5} d^{-18 / 5}\left[\left(\int_{0}^{1} u^{7} d x\right)^{1 / 5}+\left(\int_{0}^{1} v^{7} d x\right)^{1 / 5}\right]+\epsilon \int_{0}^{1} v u_{t}^{2} d x, \\
& \alpha_{23} \int_{0}^{1} v w_{t} g d x \leq \alpha_{23} d^{-1}\left|\int_{0}^{1} u v w_{t} d x\right|+\alpha_{23} d^{-1}\left|\int_{0}^{1} v^{2} w_{t} d x\right| \\
& \leq \frac{\alpha_{23}^{2}}{2 \epsilon} M_{2}^{4 / 5} d^{-18 / 5}\left[\left(\int_{0}^{1} u^{7} d x\right)^{1 / 5}+\left(\int_{0}^{1} v^{7} d x\right)^{1 / 5}\right]+\epsilon \int_{0}^{1} v w_{t}^{2} d x, \\
& \alpha_{31} \int_{0}^{1} w u_{t} h d x \leq \alpha_{31}(\alpha+k) d^{-1}\left|\int_{0}^{1} w^{2} u_{t} d x\right|+\alpha_{31} \gamma\left|\int_{0}^{1} w^{3} u_{t} d x\right| \\
& \leq \frac{(\alpha+k)^{2} \alpha_{31}^{2}}{2 \epsilon} M_{2}^{4 / 5} d^{-18 / 5}\left(\int_{0}^{1} w^{7} d x\right)^{1 / 5} \\
& +\frac{\alpha_{31}^{2}}{2 \epsilon} \gamma^{2} M_{2}^{2 / 5} d^{-4 / 5}\left(\int_{0}^{1} w^{7} d x\right)^{3 / 5}+\epsilon \int_{0}^{1} w u_{t}^{2} d x, \\
& \alpha_{32} \int_{0}^{1} w v_{t} h d x \leq \alpha_{32}(\alpha+k) d^{-1}\left|\int_{0}^{1} w^{2} v_{t} d x\right|+\alpha_{32} \gamma\left|\int_{0}^{1} w^{3} v_{t} d x\right| \\
& \leq \frac{\left(\alpha^{2}+k^{2}\right) \alpha_{32}^{2}}{2 \epsilon} M_{2}^{4 / 5} d^{-18 / 5}\left(\int_{0}^{1} w^{7} d x\right)^{1 / 5} \\
& +\frac{\alpha_{32}^{2} \gamma^{2}}{2 \epsilon} M_{2}^{2 / 5} d^{-4 / 5}\left(\int_{0}^{1} w^{7} d x\right)^{3 / 5}+\epsilon \int_{0}^{1} w v_{t}^{2} d x .
\end{aligned}
$$


Thus

$$
\begin{aligned}
\int_{0}^{1}(1+ & \left.2 \alpha_{11}+\alpha_{12} v+\alpha_{13}\right) u_{t} f d x+\int_{0}^{1}\left(1+\alpha_{21} u+2 \alpha_{22} v+\alpha_{23} w\right) v_{t} g d x \\
& +\int_{0}^{1}\left(\eta+\alpha_{31} u+\alpha_{32} v+2 \alpha_{33} w\right) w_{t} h d x+\int_{0}^{1} \alpha_{12} u v_{t} f d x+\int_{0}^{1} \alpha_{13} u w_{t} f d x \\
& +\int_{0}^{1} \alpha_{21} v u_{t} g d x+\int_{0}^{1} \alpha_{23} v w_{t} g d x+\int_{0}^{1} \alpha_{31} w u_{t} h d x+\int_{0}^{1} \alpha_{32} w v_{t} h d x \\
\leq & \lambda \epsilon \int_{0}^{1}(u+v+w)\left(u_{t}^{2}+v_{t}^{2}+w_{t}^{2}\right) d x+\frac{C_{9}}{\epsilon} M_{1} d^{-3}\left(2+\eta^{2}\right) \\
& +\frac{C_{10}}{\epsilon} M_{2}^{4 / 5} d^{-8 / 5}\left(2+d^{-2}+\eta^{2}\right)\left[\int_{0}^{1}\left(u^{7}+v^{7}+w^{7}\right) d x\right]^{1 / 5} \\
& +\frac{C_{11}}{\epsilon} M_{2}^{4 / 5} d^{-8 / 5}\left(1+d^{2}\right)\left[\int_{0}^{1}\left(u^{7}+v^{7}+w^{7}\right) d x\right]^{3 / 5} \\
& +\frac{C_{12}}{\epsilon}\left(1+d^{2}\right) \int_{0}^{1}\left(u^{7}+v^{7}+w^{7}\right) d x
\end{aligned}
$$

where $\lambda$ is a positive constant.

Note by (2.8) and $(2.9)$ that $|P|_{7 / 2}^{7 / 2} \leq C\left(\left|P_{x}\right|_{2}^{5 / 3}|P|_{1}^{11 / 6}+|P|_{1}^{7 / 2}\right),\left|P_{x}\right|_{2}^{10 / 3} \leq$ $B_{1} K_{1}^{4 / 3} d^{-4 / 3}\left(\left|P_{x x}\right|_{2}^{2}+K_{1}^{2} d^{-2}\right)$, and

$$
-\frac{1}{2} \int_{0}^{1} P_{x x}^{2} d x-\frac{1}{2} \int_{0}^{1} Q_{x x}^{2} d x-\frac{\eta}{2} \int_{0}^{1} R_{x x}^{2} d x \leq-B_{2} \min \{1, \eta\} K_{1}^{-4 / 3} d^{4 / 3} \bar{y}^{5 / 3}+K_{1}^{2} d^{-2}(2+\eta) .
$$

Choose a small enough number $\epsilon>0$, such that $\lambda \epsilon<C_{3}$. According to (2.28)-(2.34), we have

$$
\begin{aligned}
\frac{1}{2} y^{\prime}(t) \leq & -A_{1} \min \{1, \eta\} K_{1}^{-4 / 3} y^{5 / 3}+A_{2} K_{2} y^{1 / 6}+A_{3} K_{3} y^{1 / 3}+A_{4} K_{4} y^{1 / 2} \\
& +A_{5} K_{5} y^{2 / 3}+A_{6} K_{6} y^{5 / 6}+A_{7} K_{7},
\end{aligned}
$$

where $y=\int_{0}^{1}\left[\left(d P_{x}\right)^{2}+\left(d Q_{x}\right)^{2}+\left(d R_{x}\right)^{2}\right] d x$.

However, (2.35) implies that there exist positive constants $\widetilde{\tau}_{3}>0$ and $\widetilde{M}_{3}$ depending on $d, d_{3}, \alpha_{i j}(i, j=1,2,3), \beta, a, b, c, k, \gamma, \alpha$, such that

$$
\int_{0}^{1}\left(d P_{x}\right)^{2} d x, \int_{0}^{1}\left(d Q_{x}\right)^{2} d x, \int_{0}^{1}\left(d R_{x}\right)^{2} d x \leq \widetilde{M}_{3}, \quad t \geq \widetilde{\tau}_{3} .
$$


When $d, d_{3} \geq 1, \eta \in[\underline{d}, \bar{d}]$, the coefficients of (2.35) can be estimated by constants depending on $\underline{d}, \bar{d}$, but not on $d, d_{3}$. Thus, when $d, d_{3} \geq 1, \eta \in[\underline{d}, \bar{d}], \widetilde{M}_{3}$ depends on $\alpha_{i j}(i, j=$ $1,2,3), \beta, a, b, c, k, \gamma, \alpha, \underline{d}, \bar{d}$, and is irrelevant to $d, d_{3} \geq 1$. Since

$$
\left(\begin{array}{c}
P_{x} \\
Q_{x} \\
R_{x}
\end{array}\right)=\left(\begin{array}{lll}
P_{u} & P_{v} & P_{w} \\
Q_{u} & Q_{v} & Q_{w} \\
R_{u} & R_{v} & R_{w}
\end{array}\right)\left(\begin{array}{c}
u_{x} \\
v_{x} \\
w_{x}
\end{array}\right),
$$

similar to (2.26) in [24], we have

$$
\left|d u_{x}\right|+\left|d v_{x}\right|+\left|d w_{x}\right| \leq D\left(\left|d P_{x}\right|+\left|d Q_{x}\right|+\left|d R_{x}\right|\right), \quad 0<x<1, t>0,
$$

where $D$ is a positive constant only depending on $\eta, \alpha_{i j}(i, j=1,2,3)$. Scaling back with (2.22) to original variable $u, v, w, t$ and combining (2.36), (2.38), there exist positive constants $\tau_{3}>0$ and $M_{3}$ depending on $d, d_{3}, \alpha_{i j}(i, j=1,2,3), \beta, a, b, c, k, \gamma, \alpha$, such that

$$
\int_{0}^{1} u_{x}^{2} d x, \int_{0}^{1} v_{x}^{2} d x, \int_{0}^{1} w_{x}^{2} d x \leq M_{3}, \quad t \geq \tau_{3}
$$

In addition, when $d, d_{3} \geq 1, \eta \in[\underline{d}, \bar{d}], M_{3}$ is dependent of $\underline{d}, \bar{d}$, but independent of $d, d_{3} \geq 1$.

(ii) It holds that $t \geq 0$. Replacing $M_{1}, M_{2}$ with $M_{1}^{\prime}, M_{2}^{\prime}$ in (2.24)-(2.34), we can obtain that there exists a positive constant $M_{3}^{\prime}$ depending on $d, d_{3}, \alpha_{i j}(i, j=1,2,3), \beta, a, b, c, k, \gamma, \alpha$ and the $W_{2}^{1}$-norm of $u_{0}, v_{0}, w_{0}$ such that

$$
\int_{0}^{1} u_{x}^{2} d x, \int_{0}^{1} v_{x}^{2} d x, \int_{0}^{1} w_{x}^{2} d x \leq M_{3}^{\prime}, \quad t \geq 0
$$

When $d, d_{3} \geq 1, \eta \in[\underline{d}, \bar{d}], M_{3}^{\prime}$ is dependent of $\underline{d}, \bar{d}$, but independent of $d, d_{3} \geq 1$.

Concluding from (2.15), (2.22), (2.39), and Sobolev embedding theorem, there exists a positive constants $t_{0}>0, M, M^{\prime}$ depending on $d, d_{3}, \alpha_{i j}(i, j=1,2,3), \beta, a, b, c, k, \gamma, \alpha$, such that (2.3) and (2.4) are satisfied. Furthermore, when $d, d_{3} \geq 1, \eta \in[\underline{d}, \bar{d}]$ and the time $t$ is large enough, $M, M^{\prime}$ are dependent of $\alpha_{i j}(i, j=1,2,3), \beta, a, b, c, k, \gamma, \alpha, \underline{d}, \bar{d}$, but independent of $d, d_{3} \geq 1$.

Similarly, according to $\left(2.15^{\prime}\right),\left(2.22^{\prime}\right),\left(2.39^{\prime}\right)$, we can see that there exists a positive constant $M^{\prime \prime}$ depending on $d, d_{3}, \alpha_{i j}(i, j=1,2,3), \beta, a, b, c, k, \gamma, \alpha$ and the initial functions $u_{0}, v_{0}, w_{0}$, such that

$$
|u(\cdot, t)|_{1,2},|v(\cdot, t)|_{1,2},|w(\cdot, t)|_{1,2} \leq M^{\prime \prime}, \quad t \geq 0
$$


When $d, d_{3} \geq 1, \eta \in[\underline{d}, \bar{d}], M^{\prime \prime}$ is dependent of $\underline{d}, \bar{d}$, but independent of $d, d_{3}$. Thus $T=+\infty$. This completes proof of Theorem 2.1.

\section{Global Stability}

From [1], we know that if

$$
\begin{gathered}
\alpha>k, \quad \beta>a, \quad \sqrt{\frac{k}{\alpha-k}}<m_{0}, \\
\frac{\beta-a-c}{2}+\frac{b^{2}}{8 c} \leq \frac{b \sqrt{p_{1}}}{24 c}+\frac{24(\beta-a) c^{2}}{3 b^{2}+4 c(\beta-a-c)-b \sqrt{p_{1}}},
\end{gathered}
$$

where $\left(p_{1}=9 b^{2}+24 c(\beta-a-c) \geq 0\right)$, then (1.1) has the unique position equilibrium point $E^{*}\left(u^{*}, v^{*}, w^{*}\right)$.

Theorem 3.1. Assume that all conditions in Theorem 2.1 and $(H)$ are satisfied. Assume further that

$$
\begin{aligned}
\frac{1}{\beta}\left(a+b u^{*}+c u^{* 2}\right)> & 2+\frac{\left(u^{* 2}+\sqrt{1+u^{* 2}}\right)^{2}}{8}+\frac{u^{* 4}}{2}, \quad \frac{\gamma}{\alpha}>\frac{1}{2\left(1+u^{* 2}\right)^{2}} \\
\frac{4}{\alpha \beta} w^{*} d^{2} d_{3}> & \frac{1}{\beta}\left(\alpha_{23} M^{2}+\frac{1}{\alpha} \alpha_{32} w^{*}\right)^{2}\left(d+2 \alpha_{11} M+\alpha_{12} M+\alpha_{13} M\right) \\
& +\left(\frac{1}{\beta} \alpha_{13} M^{2}+\frac{1}{\alpha} \alpha_{31} w^{*}\right)^{2}\left(d+\alpha_{21} M+2 \alpha_{22} M+\alpha_{23} M\right) \\
& +\frac{1}{\alpha}\left(\frac{1}{\beta} \alpha_{12}+\alpha_{21}\right)^{2} M^{2} w^{*}\left(d_{3}+\alpha_{31} M+\alpha_{32} M+2 \alpha_{33} M\right)
\end{aligned}
$$

hold, where $M$ is the positive constant in (2.4). Then the unique positive equilibrium point $E^{*}$ of (1.1) is globally asymptotically stable.

Remark 3.2. Since $M$ is independent of $d_{1} d_{3}$ in the case of $d_{,} d_{3} \geq 1,(3.2)$ is always satisfied if $d$ and $d_{3}$ are big enough.

Proof. Define the Lyapunov function

$$
H(u, v, w)=\frac{1}{2 \beta} \int_{0}^{1}\left(u-u^{*}\right)^{2} d x+\frac{1}{2} \int_{0}^{1}\left(v-v^{*}\right)^{2} d x+\frac{1}{\alpha} \int_{0}^{1}\left(w-w^{*}-w^{*} \ln \frac{w}{w^{*}}\right) d x .
$$


Let $(u, v, w)$ be any solution of (1.1) with initial functions $u_{0}(x), v_{0}(x), w_{0}(x) \geq(\not \equiv) 0$. From the strong maximum principle for parabolic equations, it is not hard to verify that $u, v, w>0$ for $t>0$. Thus

$$
\begin{aligned}
\frac{d H}{d t} \leq-\int_{0}^{1}\left[\frac{1}{\beta}\left(d+2 \alpha_{11} u+\alpha_{12} v+\alpha_{13} w\right) u_{x}^{2}+\left(d+\alpha_{21} u+2 \alpha_{22} v+\alpha_{23} w\right) v_{x}^{2}\right. \\
+\frac{1}{\alpha}\left(d_{3}+\alpha_{31} u+\alpha_{32} v+2 \alpha_{33} w\right) \frac{w^{*}}{w^{2}} w_{x}^{2}+\left(\frac{1}{\beta} \alpha_{12} u+\alpha_{21} v\right) u_{x} v_{x} \\
\left.+\left(\frac{1}{\beta} \alpha_{13} u+\frac{1}{\alpha} \alpha_{31} \frac{w^{*}}{w}\right) u_{x} w_{x}+\left(\alpha_{23} v+\frac{1}{\alpha} \alpha_{32} \frac{w^{*}}{w}\right) v_{x} w_{x}\right] d x \\
-\int_{0}^{1}\left\{\left(u-u^{*}\right)^{2} \frac{1}{\beta}\left[a+b\left(u+u^{*}\right)+c\left(u^{2}+u u^{*}+u^{* 2}\right)+\frac{w\left(u+u^{*}\right)}{\left(1+u^{2}\right)\left(1+u^{* 2}\right)}\right]\right. \\
\left.-2-\frac{1}{2}\left(\frac{u+u^{*}}{1+u^{2}}-u^{* 2}\right)^{2}\right\} d x-\frac{1}{2} \int_{0}^{1}\left(v-v^{*}\right)^{2} d x \\
-\int_{0}^{1}\left(w-w^{*}\right)^{2}\left[\frac{\gamma}{\alpha}-\frac{1}{2\left(1+u^{* 2}\right)^{2}}\right] d x
\end{aligned}
$$

The first integrand in the right hand of the above inequality is positive definite if

$$
\begin{aligned}
\frac{4}{\alpha \beta} w^{*}( & \left.d+2 \alpha_{11} u+\alpha_{12} v+\alpha_{13} w\right)\left(d+\alpha_{21} u+2 \alpha_{22} v+\alpha_{23} w\right)\left(d_{3}+\alpha_{31} u+\alpha_{32} v+2 \alpha_{33} w\right) \\
& +w^{2}\left(\frac{1}{\beta} \alpha_{12} u+\alpha_{21} v\right)\left(\frac{1}{\alpha} \alpha_{13} u+\frac{1}{\alpha} \alpha_{31} \frac{w^{*}}{w}\right)\left(\alpha_{23} v+\beta \alpha_{32} \frac{w^{*}}{w}\right) \\
> & \frac{1}{\beta}\left(\alpha_{23} v w+\frac{1}{\alpha} \alpha_{32} w^{*}\right)^{2}\left(d+2 \alpha_{11} u+\alpha_{12} v+\alpha_{13} w\right) \\
& +\left(\frac{1}{\beta} \alpha_{13} u w+\frac{1}{\alpha} \alpha_{31} w^{*}\right)^{2}\left(d+\alpha_{21} u+2 \alpha_{22} v+\alpha_{23} w\right) \\
& +\frac{1}{\alpha} w^{*}\left(\frac{1}{\beta} \alpha_{12} u+\alpha_{21} v\right)^{2}\left(d_{3}+\alpha_{31} u+\alpha_{32} v+2 \alpha_{33} w\right) .
\end{aligned}
$$

From the maximum-norm estimate in Theorem 2.1, (3.2) is a sufficient condition of (3.5). Thus when (3.1) holds, there exists a positive constant $\delta$ such that

$$
\frac{d H(u, v, w)}{d t} \leq-\delta \int_{0}^{1}\left[\left(u-u^{*}\right)^{2}+\left(v-v^{*}\right)^{2}+\left(w-w^{*}\right)^{2}\right] d x
$$


By integration by parts, Hölder inequality and the maximum-norm estimate in Theorem 2.1, we can see that $d / d t\left(\int_{0}^{1}\left[\left(u-u^{*}\right)^{2}+\left(v-v^{*}\right)^{2}+\left(w-w^{*}\right)^{2}\right]\right) d x$ is bounded from above. According to Lemma 3.1 in [1] and (3.6), we obtain

$$
\left|u(\cdot, t)-u^{*}\right|_{2} \longrightarrow 0, \quad\left|v(\cdot, t)-v^{*}\right|_{2} \longrightarrow 0, \quad\left|w(\cdot, t)-w^{*}\right|_{2} \longrightarrow 0, \quad(t \longrightarrow \infty)
$$

Using Gagliardo-Nirenberg inequalities, we have $|u(\cdot, t)|_{\infty} \leq C|u|_{1,2}^{1 / 2}|u|_{2}^{1 / 2}$. Thus

$$
\left|u(\cdot, t)-u^{*}\right|_{\infty} \longrightarrow 0, \quad\left|v(\cdot, t)-v^{*}\right|_{\infty} \longrightarrow 0, \quad\left|w(\cdot, t)-w^{*}\right|_{\infty} \longrightarrow 0, \quad(t \longrightarrow \infty)
$$

That is, $(u, v, w)$ converges uniformly to $E^{*}$. Since $H(u, v, w)$ is decreasing for $t>0, E^{*}$ is globally asymptotically stable.

\section{Acknowledgments}

This work has been partially supported by the China National Natural Science Foundation (no. 10871160), the NSF of Gansu Province (no. 096RJZA118), the Scientific Research Fund of Gansu Provincial Education Department, and the NWNU-KJCXGC-03-47 Foundation.

\section{References}

[1] R. Zhang, L. Guo, and S. Fu, "Global behavior for a diffusive predator-prey model with stage structure and nonlinear density restriction-I: the case in $\mathbb{R}^{n}$," Boundary Value Problems, vol. 2009, Article ID 378763, p. 27, 2009.

[2] J. Blat and K. J. Brown, "Bifurcation of steady-state solutions in predator-prey and competition systems," Proceedings of the Royal Society of Edinburgh, vol. 97, pp. 21-34, 1984.

[3] J. Blat and K. J. Brown, "Global bifurcation of positive solutions in some systems of elliptic equations," SIAM Journal on Mathematical Analysis, vol. 17, no. 6, pp. 1339-1353, 1986.

[4] J. C. Eilbeck, J. E. Furter, and J. López-Gómez, "Coexistence in the competition model with diffusion," Journal of Differential Equations, vol. 107, no. 1, pp. 96-139, 1994.

[5] E. N. Dancer, "On positive solutions of some pairs of differential equations," Transactions of the American Mathematical Society, vol. 284, no. 2, pp. 729-743, 1984.

[6] E. N. Dancer, "On positive solutions of some pairs of differential equations. II," Journal of Differential Equations, vol. 60, no. 2, pp. 236-258, 1985.

[7] E. N. Dancer, J. López-Gómez, and R. Ortega, “On the spectrum of some linear noncooperative elliptic systems with radial symmetry," Differential and Integral Equations, vol. 8, no. 3, pp. 515-523, 1995.

[8] Y. Du and Y. Lou, "Some uniqueness and exact multiplicity results for a predator-prey model," Transactions of the American Mathematical Society, vol. 349, no. 6, pp. 2443-2475, 1997.

[9] Y. Du and Y. Lou, "S-shaped global bifurcation curve and Hopf bifurcation of positive solutions to a predator-prey model," Journal of Differential Equations, vol. 144, no. 2, pp. 390-440, 1998.

[10] Y. Du and Y. Lou, "Qualitative behaviour of positive solutions of a predator-prey model: effects of saturation," Proceedings of the Royal Society of Edinburgh, vol. 131, no. 2, pp. 321-349, 2001.

[11] J. López-Gomez and R. M. Pardo, "Coexistence in a simple food chain with diffusion," Journal of Mathematical Biology, vol. 30, no. 7, pp. 655-668, 1992.

[12] J. López-Gómez and R. M. Pardo, "Existence and uniqueness of coexistence states for the predatorprey model with diffusion: the scalar case," Differential and Integral Equations, vol. 6, no. 5, pp. 10251031, 1993.

[13] J. López-Gómez and R. M. Pardo, "Invertibility of linear noncooperative elliptic systems," Nonlinear Analysis: Theory, Methods E Applications, vol. 31, no. 5-6, pp. 687-699, 1998.

[14] K. Nakashima and Y. Yamada, "Positive steady states for prey-predator models with cross-diffusion," Advances in Differential Equations, vol. 1, no. 6, pp. 1099-1122, 1996. 
[15] H. Zhang, P. Georgescu, and L. Chen, "An impulsive predator-prey system with BeddingtonDeAngelis functional response and time delay," International Journal of Biomathematics, vol. 1, no. 1, pp. 1-17, 2008.

[16] Y. Fan, L. Wang, and M. Wang, "Notes on multiple bifurcations in a delayed predator-prey model with nonmonotonic functional response," International Journal of Biomathematics, vol. 2, no. 2, pp. 129-138, 2009.

[17] F. Wang and Y. An, "Existence of nontrivial solution for a nonlocal elliptic equation with nonlinear boundary condition," Boundary Value Problems, vol. 2009, Article ID 540360, 8 pages, 2009.

[18] S. Shim, "Uniform boundedness and convergence of solutions to cross-diffusion systems," Journal of Differential Equations, vol. 185, no. 1, pp. 281-305, 2002.

[19] S. Shim, "Uniform boundedness and convergence of solutions to the systems with cross-diffusions dominated by self-diffusions," Nonlinear Analysis: Real World Applications, vol. 4, no. 1, pp. 65-86, 2003.

[20] S. Shim, "Uniform boundedness and convergence of solutions to the systems with a single nonzero cross-diffusion," Journal of Mathematical Analysis and Applications, vol. 279, no. 1, pp. 1-21, 2003.

[21] M. Wang, Nonliear Parabolic Equation of Parabolic Type, Science Press, Beijing, China, 1993.

[22] H. Amann, "Dynamic theory of quasilinear parabolic equations-II: reaction-diffusion systems," Differential and Integral Equations, vol. 3, no. 1, pp. 13-75, 1990.

[23] H. Amann, "Dynamic theory of quasilinear parabolic systems-III: global existence," Mathematische Zeitschrift, vol. 202, no. 2, pp. 219-250, 1989.

[24] S. Fu, Z. Wen, and S. Cui, "Uniform boundedness and stability of global solutions in a strongly coupled three-species cooperating model," Nonlinear Analysis: Real World Applications, vol. 9, no. 2, pp. 272-289, 2008. 